\begin{tabular}{|c|l|}
\hline Title & $\begin{array}{l}\text { Fabrication and characterization of a GaA s based three terminal nanowire junction device controlled by double } \\
\text { Schottky wrap gates }\end{array}$ \\
\hline Author(s) & Nakamura, Tatsuya; Kasai, Seiya; Shiratori, Y Yta; Hashizume, Tamotsu \\
\hline Citation & $\begin{array}{l}\text { A pplied Physics Letters, 90(10), 102104 } \\
\text { https://doi.org/L0.1063/2711374 }\end{array}$ \\
\hline Issue Date & 2007-03 \\
\hline Doc URL & http://hdl.handle.net/2115/20120 \\
\hline Type & article \\
\hline Note & Copyright $\odot 2007$ A merican Institute of Physics \\
\hline File Information & APL90.pdf \\
\hline
\end{tabular}

Instructions for use 


\title{
Fabrication and characterization of a GaAs-based three-terminal nanowire junction device controlled by double Schottky wrap gates
}

\author{
Tatsuya Nakamura, ${ }^{\text {a) }}$ Seiya Kasai, ${ }^{\text {b) }}$ Yuta Shiratori, and Tamotsu Hashizume \\ Research Center for Integrated Quantum Electronics, Hokkaido University, North 13, West 8, Kita-ku, \\ Sapporo 060-8628, Japan and Graduate School of Information Science and Technology, Hokkaido University, \\ North 14, West 9, Kita-ku, Sapporo 060-0814, Japan
}

(Received 19 December 2006; accepted 29 January 2007; published online 6 March 2007)

\begin{abstract}
A three-terminal nanowire junction device controlled by double nanometer-sized Schottky wrap gates (WPGs), which control left and right branches independently, are fabricated utilizing AlGaAs/GaAs etched nanowires and characterized experimentally. Fabricated device exhibits clear nonlinear characteristics of output voltage at the center terminal by applying voltages to left and right terminals in push-pull fashion. Applying asymmetric gate voltages to left and right WPGs provides clear asymmetry in the output voltage. The nonlinearity in the low voltage regions is greatly enhanced by squeezing both left and right branches using WPGs. (C) 2007 American Institute of Physics. [DOI: 10.1063/1.2711374]
\end{abstract}

Recently, nanowires have attracted much attention for the next-generation nanodevice materials. Nanowire junctions are important building blocks for nanowire-based devices and their integrated circuits. Rich functionalities were expected theoretically in these systems due to the ballistic transport of carriers ${ }^{1}$ and self gating. ${ }^{2}$ Clear nonlinear characteristics have been observed in three-terminal nanowire junctions of III-V compound semiconductors ${ }^{3,4}$ and carbon nanotubes, ${ }^{5}$ even at room temperature. In addition, capability of their high-speed operation has been demonstrated experimentally. ${ }^{4,6}$ Their application to logic circuits has been also intensively investigated. ${ }^{7,8}$ In this letter, we fabricate and characterize a GaAs-based three-terminal nanowire junction device, in which two branches can be controlled independently by nanometer-sized Schottky wrap gates (WPGs), in order to control the nonlinear characteristics toward nanowire-based circuits and systems.

Figure 1(a) shows a scanning electron microscopy (SEM) image of a fabricated device. The device had a T-branch nanowire junction formed on an AlGaAs/GaAs heterostructure wafer by electron beam lithography and wet chemical etching. Geometrical nanowire width $W_{\text {geo }}$ was $560 \mathrm{~nm}$. Nanowire lengths for left and right branches were $3 \mu \mathrm{m}$ and that for a center branch was $2.6 \mu \mathrm{m}$. $\mathrm{Cr} / \mathrm{Au}$ Schottky WPGs of $400 \mathrm{~nm}$ gate lengths were formed on the left and right branches. The WPG wrapped around a nanowire can squeeze effective nanowire width $W_{\text {eff }}$, and onedimensional channel is formed under suitable gate voltage. ${ }^{9}$ Measured mobility and the sheet carrier density of the unprocessed wafer at room temperature (RT) were $7100 \mathrm{~cm}^{2} / \mathrm{V} \mathrm{s}$ and $7.8 \times 10^{11} \mathrm{~cm}^{-2}$, respectively. WPGcontrolled nanowire branches of the fabricated device could operate as conventional field effect transistors, as shown in Fig. 1(b). They showed good gate control characteristics and operated similarly to each other. Their threshold voltages for nanowire channel pinch off were $-0.9 \mathrm{~V}$. In this study, all measurements were carried out at RT.

\footnotetext{
a)Electronic mail: nakamura@ rciqe.hokudai.ac.jp

b)Electronic mail: kasai@rciqe.hokudai.ac.jp
}

Figure 2 shows measured output voltages at the center terminal, $V_{\mathrm{C}}$, by applying voltages to the left and right terminals, $V_{\mathrm{L}}$ and $V_{\mathrm{R}}$, respectively, in push-pull fashion, namely, $V_{\mathrm{R}}=-V_{\mathrm{L}}$. When left and right WPG voltages $V_{\mathrm{GL}}$ and $V_{\mathrm{GR}}$, respectively, were kept at $0 \mathrm{~V}, V_{\mathrm{C}}$ showed bell-like curves and was always negative as shown in Fig. 2, indicated by dashed lines. We also fabricated and characterized a three-terminal device without WPGs and similar characteristic was obtained. Next, $V_{\mathrm{C}}$ was measured by changing $V_{\mathrm{GL}}$ while keeping $V_{\mathrm{GR}}$ at $0 \mathrm{~V}$. Obtained $V_{\mathrm{C}}-V_{\mathrm{L}}$ curves are shown in Fig. 2(a). Asymmetric feature was clearly introduced by applying negative voltages to the left WPG. When $\left|V_{\mathrm{L}}\right|$

(a)
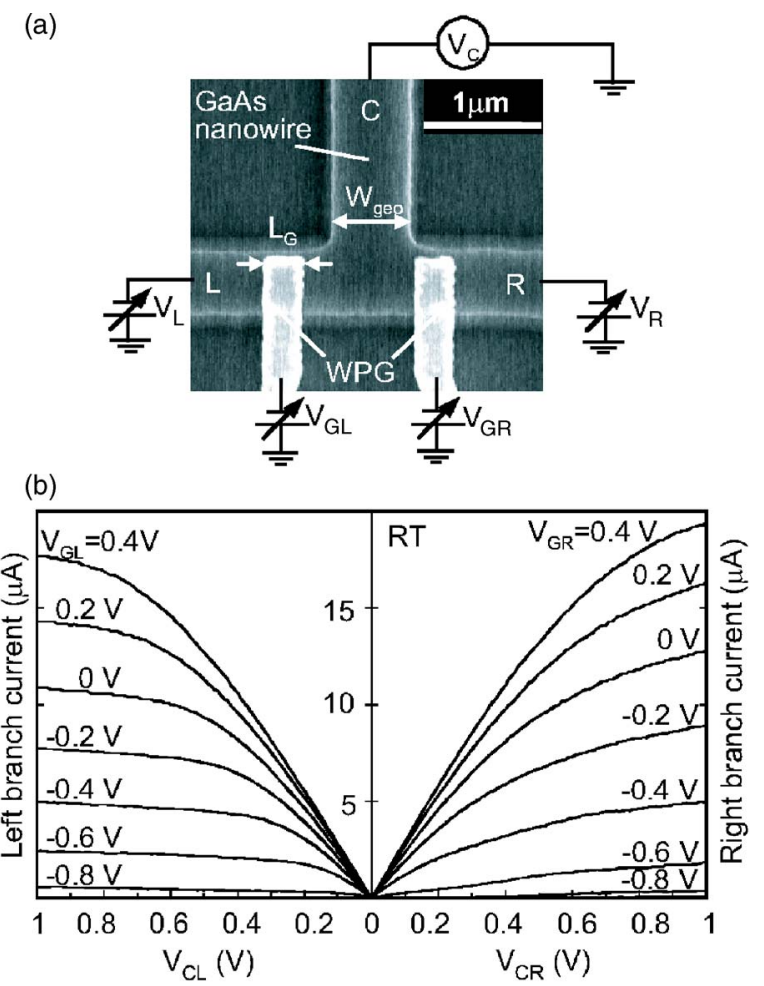

FIG. 1. (a) SEM image of a fabricated three-terminal nanowire junction device with double WPGs and (b) $I-V$ characteristics for left and right branches controlled by WPGs. 

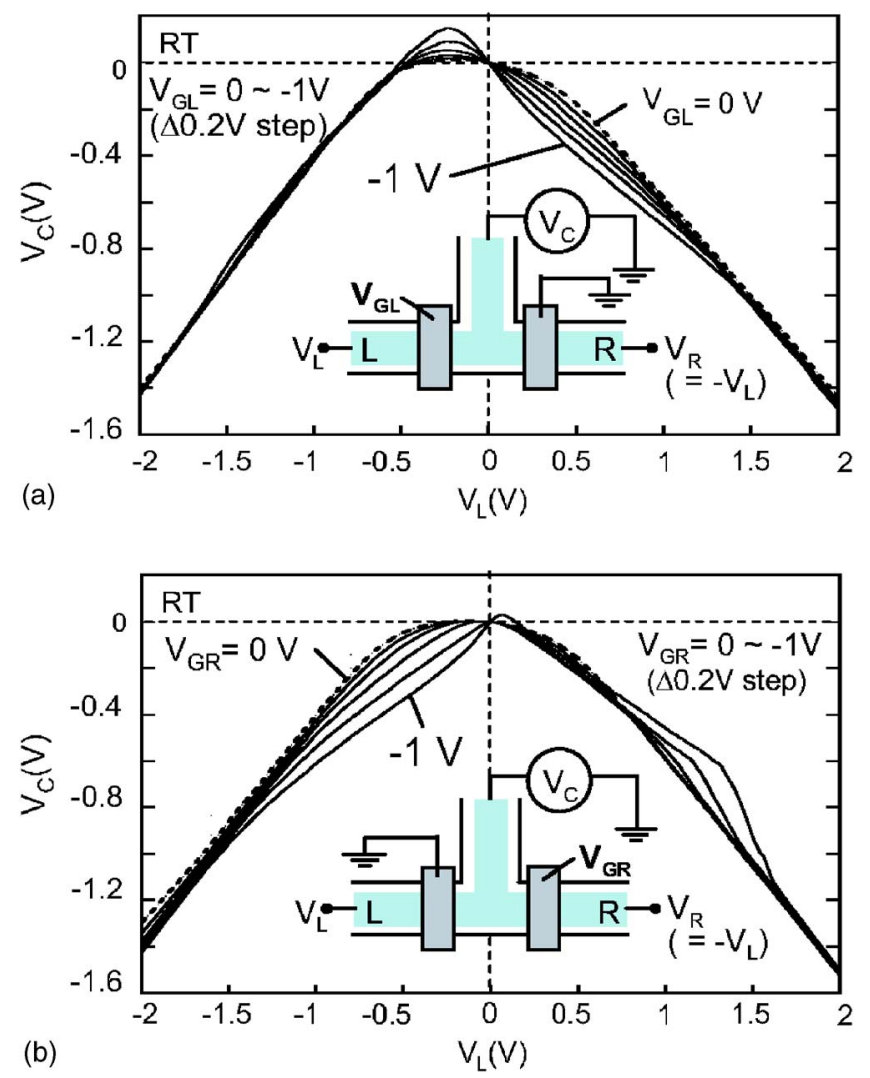

FIG. 2. Output voltage at the center terminal as a function of the left terminal voltage for changing (a) left and (b) right hand side WPG voltages, respectively. Here, $V$ and $-V$ are applied to the left and right terminals in push-pull fashion. The insets show measurement setups.

$<0.6 \mathrm{~V}$, the asymmetry became remarkable as decrease of $V_{\mathrm{GL}}$. Squeezing the left branch by further decreasing $V_{\mathrm{GL}}$ to the threshold voltage, peak positions of $V_{\mathrm{C}}$ curves slightly shifted toward negative. At the same time, the top of the peaks increased and became positive. Observed asymmetric characteristics were clearer than those in previously reported devices with side gates ${ }^{10}$ or top gates. ${ }^{3}$ When $\left|V_{\mathrm{L}}\right|>0.6 \mathrm{~V}$, $V_{\mathrm{C}}$ turned to hardly depend on the left WPG voltage and it remained almost same to the curve at $V_{\mathrm{GL}}=0 \mathrm{~V}$. Similar behaviors were obtained by changing $V_{\mathrm{GR}}$ while keeping $V_{\mathrm{GL}}$ at $0 \mathrm{~V}$, as shown in Fig. 2(b), however, left and right sides of the curves were reversed symmetrically. Observed $V_{\mathrm{C}}$ peak heights in Fig. 2(b) were a little bit smaller than those in Fig. 2(a), which seemed due to unintentionally slight shift of WPGs alignment, as shown in Fig. 1(a).

In the case of $V_{\mathrm{GL}}=V_{\mathrm{GR}}=0 \mathrm{~V}$, the parabolic curves in the low voltage region was similar to those in the ballistic transport domain as explained by $\mathrm{Xu} .{ }^{1}$ The mean free path of electron, $l_{e}$, in the present device was $100 \mathrm{~nm}$ and it was shorter than the geometrical junction size. However, devices with wide nanowire width of $720 \mathrm{~nm}$ were tested and the nonlinearity of $V_{\mathrm{C}}$ was found weakened, indicating that our devices still had size dependence of the nonlinear characteristics. To clarify the mechanism of the observed nonlinearity, it is necessary to characterize the operation as a function of $l_{e}$ by decreasing temperature from diffusive to ballistic transport regime. It is now under preparation.

Increasing $\left|V_{\mathrm{L}}\right|$ larger than $0.6 \mathrm{~V}, V_{\mathrm{C}}$ curves became almost linear. This behavior was also reported by Mateos et al., ${ }^{11}$ and was explained by the concentration of electric field and decrease of conductance in the positively biased branch Downloaded 19 Mar 2007 to 133.87.26.100. Redistribution subject

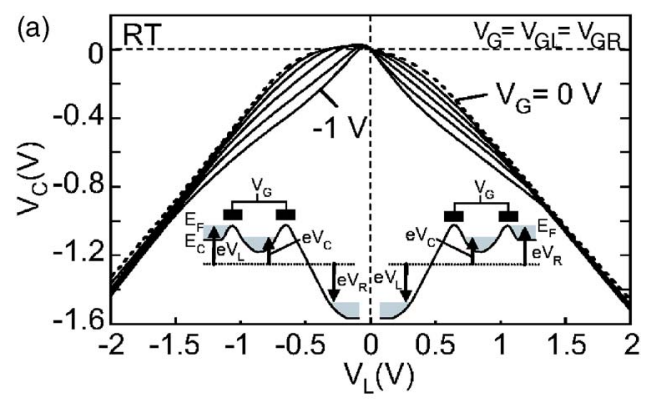

(b)

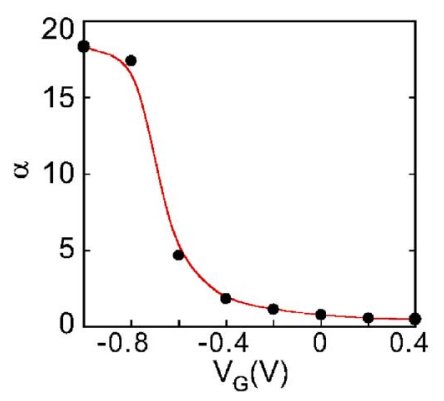

FIG. 3. (a) Output voltage at the center terminal as a function of the left terminal voltage when common gate voltage $V_{\mathrm{G}}$ was applied to two WPGs. The insets show schematics of possible potential diagrams in the junctions. (b) Evaluated curvature $\alpha$ as a function of WPG voltage.

due to intervalley scattering of electrons. Experimentally observed features for applying asymmetric WPG voltages in Fig. 2 also corresponded to the theoretical prediction by $\mathrm{Xu},{ }^{12}$ where larger lateral confinement potential in the left branch than that in the right branch resulted in the shift of the peak position to negative in $V_{\mathrm{L}}$ and the increase of the peak top. It is noted that $V_{\mathrm{C}}$ curves both in Figs. 2(a) and 2(b) were modulated largely by the WPG on the positively biased branch in our device. This indicated that $V_{\mathrm{C}}$ was controlled mostly by the situation in the positively biased branch. Several shoulders were seen around $V_{\mathrm{L}}=1.5 \mathrm{~V}$ in Fig. 2(b), however, they were unstable and irreproducible. The reason is not known yet.

Figure 3(a) shows output voltages as a function of $V_{\mathrm{L}}$ by applying same gate voltages to both two WPGs, $V_{\mathrm{GL}}=V_{\mathrm{GR}}$ $=V_{\mathrm{G}}$. In this operation, almost symmetric curves were obtained. When $\left|V_{\mathrm{L}}\right|<0.6 \mathrm{~V}$, curvature of the output voltage became sharper as decreasing $V_{\mathrm{G}}$. Observed curves were quite similar to those by the combination of $V_{\mathrm{C}}$ curves for $V_{\mathrm{L}}>0 \mathrm{~V}$ in Fig. 2(a) and those for $V_{\mathrm{L}}<0 \mathrm{~V}$ in Fig. 2(b). This fact again indicated that $V_{\mathrm{C}}$ was controlled by the condition in the positively biased branch. To evaluate the nonlinearity of outputs, $V_{\mathrm{C}}$ curves in the low voltage region were fitted by the theoretical curve for the ballistic regime, ${ }^{1}$ $V_{\mathrm{C}}=-\alpha V_{L}^{2} / 2$, where $\alpha$ is constant. Large $\alpha$ is preferable for device and circuit applications because sharp output curve corresponds to high voltage signal transfer efficiency. The obtained $\alpha$ is plotted as a function of WPG voltage in Fig. 3(b). It was found that $\alpha$ increased by the decrease of WPG voltage and it rapidly changed when $V_{\mathrm{G}}$ was around $-0.8 \mathrm{~V}$. $\alpha$ of 18 was obtained at $V_{\mathrm{G}}=-1 \mathrm{~V}$. This value is higher than that evaluated for the top-gate device at $200 \mathrm{~K}^{3}$. The theory ${ }^{1}$ indicates that $\alpha$ increases as the decrease of Fermi energy in the junction, $E_{F}$, by $\alpha \propto 1 / E_{F}$. Assuming that the operation of the present device obeys the theory, the obtained results are qualitatively consistent with the theory, since WPGs can decrease $E_{F}$ in the junction by lateral depletion from the gate to AIP license or copyright, see http://apl.aip.org/apl/copyright.jsp 
edges. The rapid increase of $\alpha$ around $-0.8 \mathrm{~V}$ indicates that $E_{F}$ decreases largely by the WPGs.

It should be mentioned that double WPG configuration provides another mechanism to increase $\alpha$ due to the formation of asymmetric potential barriers in the left and right branches by WPGs with same gate voltage. Voltage difference between the positively biased terminal and the WPG was $V_{\mathrm{G}}-\left|V_{\mathrm{L}}\right|$, although that for the negatively biased branch was $V_{\mathrm{G}}+\left|V_{\mathrm{L}}\right|$. Then, barrier potential under the WPG in the former is relatively larger than that in the latter, as schematically shown in the insets of Fig. 3(a). This can also explain that $V_{\mathrm{C}}$ is mostly controlled by the WPG on the positively biased branch. Because a larger barrier results in smaller conductance, $V_{\mathrm{C}}$ comes to follow the voltage in the negatively biased branch by $V_{\mathrm{C}}=\left[\left(G_{\mathrm{L}}-G_{\mathrm{R}}\right) /\left(G_{\mathrm{L}}+G_{\mathrm{R}}\right)\right] V_{\mathrm{L}}$, where $G_{\mathrm{L}}$ and $G_{\mathrm{R}}$ are conductances in left and right branches, respectively. Additionally, $V_{\mathrm{C}}$ curves still followed the potential in the negatively biased branch even when the electron channel in the positively biased branch was nearly pinched off. In this case, the observed behavior can be reasonably understood by the model that the center branch is connected with the left and right branches capacitively. Then $V_{\mathrm{C}}$ is given by $\left[\left(C_{\mathrm{L}}-C_{\mathrm{R}}\right) /\left(C_{\mathrm{L}}+C_{\mathrm{R}}\right)\right] V_{\mathrm{L}}$, where $C_{\mathrm{L}}$ and $C_{\mathrm{R}}$ are capacitances in left and right branches, respectively. Again, larger potential barrier in the positively biased branch results in smaller capacitance, resulting in $V_{\mathrm{C}}<0 \mathrm{~V}$. This effect can coexist with previously reported mechanisms for the nonlinear characteristics. Therefore, the double WPG configuration is useful to enhance nonlinear characteristics of threeterminal nanowire junction devices.

This work is supported in part by 21COE program, "Meme-media Technology Approach to the R\&D of nextgeneration ITs," Grant-in-Aid (17686028 and 18651074) from MEXT, Japan, and FY2004 Industrial Tech. Res. Grant Program from NEDO, Japan.

${ }^{1}$ H. Q. Xu, Appl. Phys. Lett. 78, 2064 (2001).

${ }^{2}$ J.-O. J. Wesström, Phys. Rev. Lett. 82, 2564 (1999).

${ }^{3}$ I. Shorubalko, H. Q. Xu, I. Maximov, P. Omiling, L. Samuelson, and W. Seifert, Appl. Phys. Lett. 79, 1384 (2001).

${ }^{4}$ L. Worschech, A. Schliemann, S. Reitzenstein, P. Hartmann, and A. Forchel, Microelectron. Eng. 63, 217 (2002).

${ }^{5}$ C. Papadopoulos, A. Rakitin, J. Li, A. S. Vedeneev, and J. M. Xu, Phys. Rev. Lett. 85, 3476 (2000).

${ }^{6}$ L. Bednarz, Rashim, B. Hackens, G. Farhi, V. Bayot, and I. Hynene, IEEE Trans. Nanotechnol. 4, 576 (2005).

${ }^{7}$ S. Reitzenstein, L. Worschech, and A. Forchel, IEEE Electron Device Lett. 24, 625 (2003).

${ }^{8}$ H. Q. Xu, I. Shorubalko, D. Wallin, I. Maximov, P. Omling, L. Samuelson, and W. Seifert, IEEE Electron Device Lett. 25, 164 (2004).

${ }^{9}$ S. Kasai, K. Jinushi, H. Tomozawa, and H. Hasegawa, Jpn. J. Appl. Phys., Part 1 36, 1678 (1997).

${ }^{10}$ L. Worschech, H. Q. Xu, A. Forchel, and L. Samuelson, Appl. Phys. Lett. 79, 3287 (2001).

${ }^{11}$ J. Mateos, B. G. Vasallo, D. Pardo, T. González, E. Pichonat, J.-S. Galloo, S. Bollaert, Y. Roelens, and A. Cappy, IEEE Electron Device Lett. 25, 235 (2004).

${ }^{12}$ H. Q. Xu, Physica E (Amsterdam) 13, 942 (2002). 